\title{
Genetic Analysis of DSCAM's Role as a Netrin-1 Receptor in Vertebrates
}

\author{
Elena Palmesino, ${ }^{1}$ Patrick C. G. Haddick, ${ }^{2}$ Marc Tessier-Lavigne, ${ }^{2,3}$ and Artur Kania ${ }^{1,4,5}$ \\ ${ }^{1}$ Institut de recherches cliniques de Montréal, Montréal, Québec, H2W 1R7, Canada, ${ }^{2}$ Division of Research, Genentech Inc., South San Francisco, California \\ 94080, ${ }^{3}$ Laboratory of Brain Development and Repair, The Rockefeller University, New York, New York 10021, ${ }^{4}$ Departement de medécine, Université de \\ Montréal, Montréal, Québec, H3C 3J7, Canada, and 5 Departments of Biology, Cell Biology and Anatomy, and Division of Experimental Medicine, McGill \\ University, Montréal, Québec, H3A 2T5, Canada
}

Down syndrome cell adhesion molecule (DSCAM) has mainly been characterized for its function as an adhesion molecule in axon growth and in self-recognition between dendrites of the same neuron. Recently, it has been shown that DSCAM can bind to Netrin-1 and that downregulation of DSCAM expression by siRNAs in chick and rodent spinal cords leads to impaired growth and turning response of commissural axons to Netrin-1. To investigate the effect of complete genetic ablation of DSCAM on Netrin-1-induced axon guidance, we analyzed spinal commissural neurons in DSCAM-null mice and found that they extend axons that reach and cross the floor plate and express apparently normal levels of the Netrin receptors DCC (deleted in colorectal carcinoma) and Neogenin. In vitro, commissural neurons in dorsal spinal cord explants of DSCAM-null embryos show normal outgrowth in response to Netrin-1. We therefore conclude that DSCAM is not required for Netrin-induced commissural axon outgrowth and guidance in mice.

\section{Introduction}

Netrin-1 and its receptor DCC (deleted in colorectal carcinoma) play an essential role in directing axons toward the midline in bilateral animals (Evans and Bashaw, 2010). Nevertheless, several lines of evidence suggest that not all of $\mathrm{Ne}$ trin's function in axon attraction is mediated by DCC (Serafini et al., 1994; Keino-Masu et al., 1996; Fazeli et al., 1997), and it was recently reported that DSCAM (Down's syndrome cell adhesion molecule) also acts as a Netrin-1 receptor (Andrews et al., 2008; Ly et al., 2008; Liu et al., 2009); however, genetic evidence implicating DSCAM in Netrin-mediated axon guidance in vertebrates is still lacking.

Vertebrate commissural (C) neurons are located in the dorsal spinal cord and project their axons toward and across the floor plate located at the ventral midline (Tessier-Lavigne et al., 1988). Netrin-1 secreted from the floor plate binds to DCC expressed on $\mathrm{C}$ axons, promoting their outgrowth and attraction to the midline. Upon midline crossing by $\mathrm{C}$ growth cones, DCC signaling is silenced and cues distinct from Netrin-1 instruct $C$ axon exit from the midline, prevent midline reentry and guide them in a rostral direction (Evans and Bashaw, 2010). Biochemical and

\footnotetext{
Received July 12, 2011; revised 0ct. 11, 2011; accepted Nov. 4, 2011.

Author contributions: E.P., P.C.G.H., and A.K. designed research; E.P. and P.C.G.H. performed research; E.P. and P.C.G.H. analyzed data; E.P., P.C.G.H., M.T.-L., and A.K. wrote the paper.

This work was supported by grants from the Canadian Institutes of Health Research (Institutes of Genetics and Neuroscience, Mental Health and Addiction) to A.K., and by Genentech (P.H. and M.T.L.). We thank N. Lamarche for the DCC antibody, J. Cardin, M. Liang, and I. Brisson for technical assistance and L. Delorme for secretarial assistance. DSCAM mice were a kind gift from Prof. Kazuhiro Yamakawa (RIKEN Brain Science Institute, Saitama, Japan).

The authors declare no competing financial interests.

Correspondence should be addressed to Artur Kania at the above address. E-mail: artur.kania@ircm.qc.ca.

D0I:10.1523/JNEUROSCI.3563-11.2012

Copyright $\odot 2012$ the authors $\quad 0270-6474 / 12 / 320411-06 \$ 15.00 / 0$
}

functional experiments in the context of $\mathrm{C}$ neurons have provided evidence that there are additional Netrin-1 receptors such as Neogenin and DSCAM, but the genetic evidence in support of such function is still lacking.

DSCAM is a conserved Ig superfamily transmembrane protein which has been implicated as a Netrin-1 receptor in both Drosophila and vertebrates (Andrews et al., 2008; Ly et al., 2008; Liu et al., 2009; Schmucker and Chen, 2009). DSCAM overexpression in Drosophila CNS neurons forces axons to cross the nervous system midline, consistent with an attractive Netrin receptor function (Andrews et al., 2008). Moreover, Drosophila triple knock-out of DSCAM, its paralog DSCAM3 and DCC homolog Frazzled results in stronger midline crossing defects than the removal of both Drosophila Netrins, suggesting that DSCAM also functions in a Netrin-independent fashion (Andrews et al., 2008).

The main evidence implicating DSCAM in Netrin signaling in vertebrates comes from in vitro experiments in which DSCAM function is blocked in cultured chick, and rodent embryonic spinal cords, resulting in impaired $\mathrm{C}$ axon extension and guidance (Ly et al., 2008; Liu et al., 2009). In vitro turning responses to Netrin-1 were also affected by DSCAM loss of function (Ly et al., 2008; Liu et al., 2009). Although these experiments include rescues of siRNA knockdown phenotypes by wild-type cDNAs, the effects of complete loss of DSCAM function in C axon outgrowth and guidance are ideally addressed by analysis of genetic null mutations.

Here we provide evidence that $\mathrm{C}$ neurons of mice lacking DSCAM do not exhibit outgrowth or guidance defects in response to Netrin-1. Furthermore, we report that compound mutants for DCC and DSCAM exhibit a commissure deficiency comparable to DCC single mutants, and therefore conclude that 

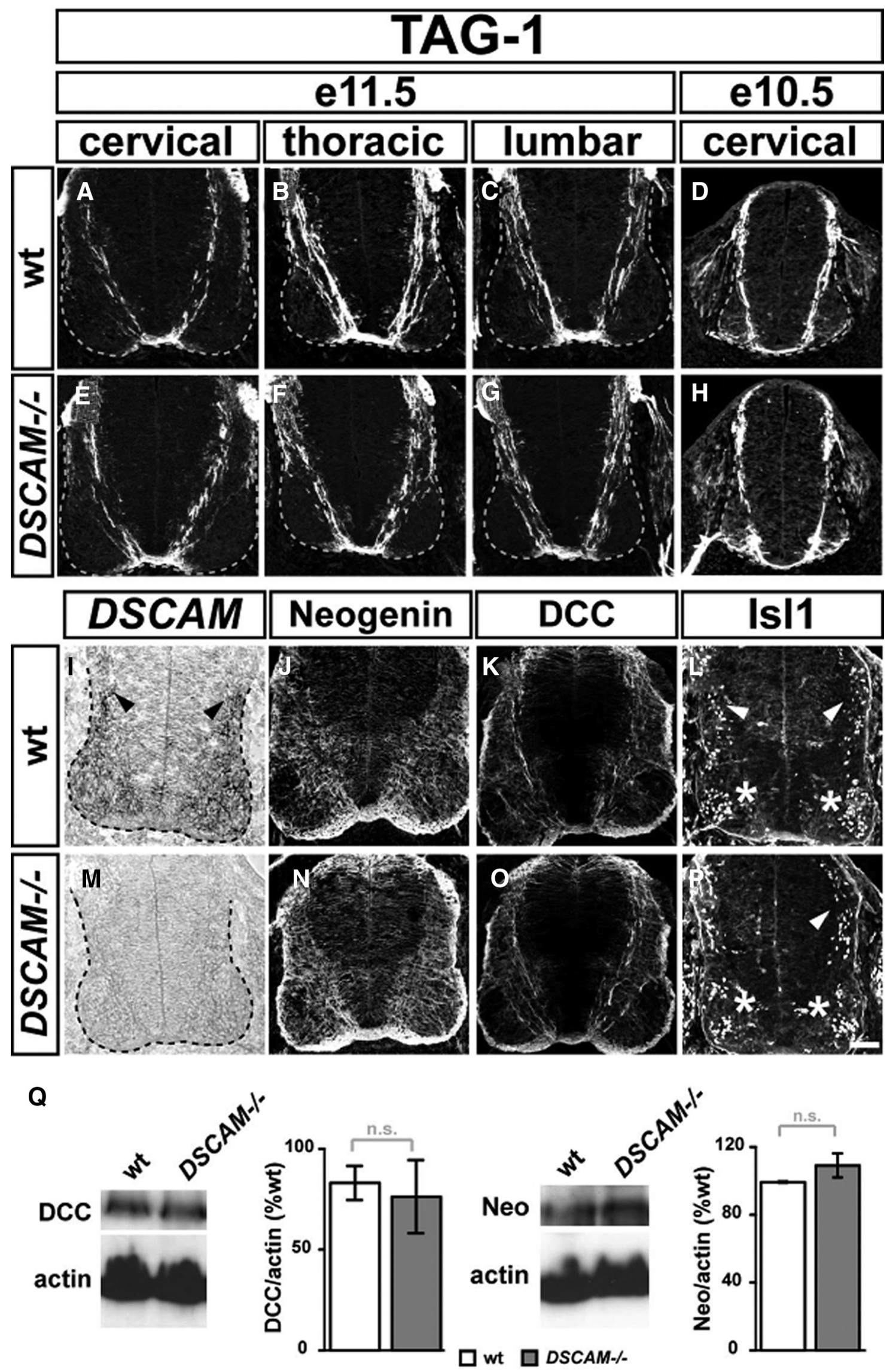

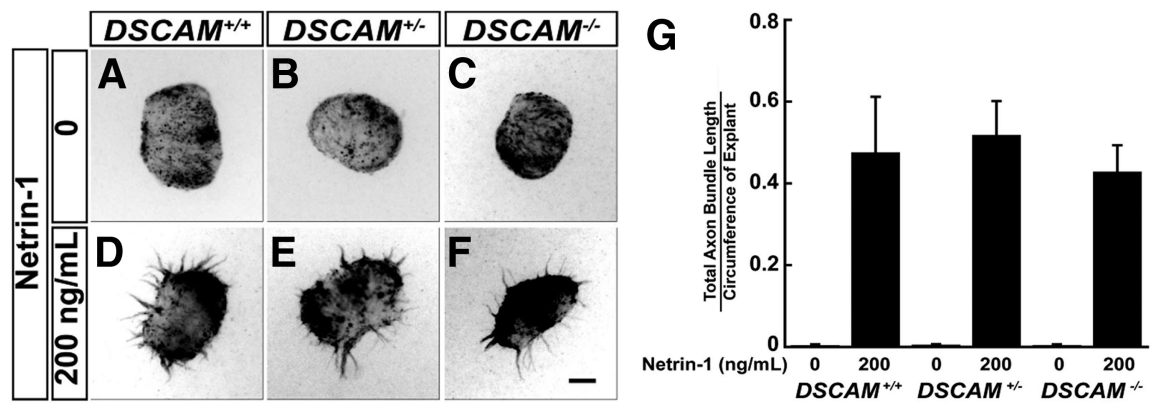

Figure 2. Netrin-induced axon outgrowth is unaffected by DSCAM mutation. $\boldsymbol{A}-\boldsymbol{F}$, Dorsal spinal cord explants of e11.5 DSCAM mutant $(\boldsymbol{C}, \boldsymbol{F}), \operatorname{DSCAM}^{+--}(\boldsymbol{B}, \boldsymbol{E})$ and control littermates $(\boldsymbol{A}, \boldsymbol{D})$ were cultured in the presence or absence of $200 \mathrm{ng} / \mathrm{ml}$ Netrin-1. G, Quantification of axon outgrowth. $N>6$ explants per condition; $n=4$ wild-type, $7 D S C A M^{+/-}$and $4 D S C A M^{-/-}$embryos. Scale bar (in $F$ ), $20 \mu$ m in all panels.

DSCAM is not required for Netrin signaling in C axon guidance in the murine spinal cord in vivo.

\section{Materials and Methods}

Animals. All mice were maintained on C57BL/6 background and genotyped by PCR as previously described (Fazeli et al., 1997; Amano et al., 2009). Embryos analyzed were of either sex.

Immunostaining and in situ $m R N A$ detection. Immunofluorescence stainings were performed on $12 \mu \mathrm{m}$ cryosections as described previously (Palmesino et al., 2010). Mouse anti TAG-1 (4D7, Developmental Studies Hybridoma Bank) was used at a dilution of 1:25, goat anti-DCC (Santa Cruz Biotechnology) was used at 1:1000, rabbit anti-Neogenin (R\&D Systems) was used at 1:100, and rabbit anti-Isl1 (kind gift from T. Jessell, Columbia University, New York, NY) was used 1:20,000 (Tsuchida et al., 1994).

In situ mRNA detections were performed as previously described (Kao et al., 2009; Palmesino et al., 2010). Probe sequence details are available upon request.

Western immunoblot. Spinal cords of e11.5 mouse embryos were lysed in $1 \%$ Triton X-100 buffer containing (in mM): 50 HEPES, pH 7.4, 150 $\mathrm{NaCl}, 10 \%$ glycerol, $1.5 \mathrm{MgCl}, 1 \mathrm{EDTA}$, protease inhibitors (Complete, Roche). Proteins were then separated by SDS-PAGE and transferred to Immobilon membrane (Millipore) followed by ECL-Western blot analysis with the following antibodies: DCC monoclonal (BD Biosciences), polyclonal Neogenin (R\&D Systems), and monoclonal $\beta$-actin (Sigma).

Dorsal spinal cord explants. The dorsal spinal cord explant assay was performed as previously described (Serafini et al., 1994) on el1.5 mouse embryos using recombinant mouse Netrin-1 (R\&D Systems).

DiI injections. Spinal cord open-book preparations from e12.5 embryos were fixed overnight in 4\% paraformaldehyde and washed in PBS. The lipophilic dye CM-DiI (Invitrogen) was injected dorsally at multiple sites along the rostro-caudal axis on one side of the open-book preparation and allowed to diffuse for $1 \mathrm{~d}$ before mounting with Mowiol and immediately followed by imaging.

Image quantification. Images were acquired using a Zeiss LSM confocal microscope or a Leica DM6000 microscope with Improvision Volocity software. Axon outgrowth and explant circumference of dorsal spinal cord explants were measured using the NeuronJ plugin for NIH ImageJ. Western blots were quantified by digitizing the exposed $\mathrm{x}$-ray film, measuring the number of signal pixels in the band and subtracting the background. Ratio of DCC or Neogenin to actin expression was then normalized to the highest wild-type value.

\section{$\leftarrow$}

(Figure legend continued.) $\quad$ DCC ( 0 ) and Neogenin ( $\boldsymbol{N}$ ) expression was maintained at levels comparable to wild-type $(\boldsymbol{J}, \boldsymbol{K})$ and position and numbers of motor neurons was not affected as revealed by $|s| 1$ immunostaining $(\boldsymbol{L}, \boldsymbol{P}) . n=4$ embryos per genotype. Arrowheads, commissural neurons; asterisks, motor neurons. Scale bar (in P), $65 \mu \mathrm{m}$ in all panels. $\boldsymbol{Q}$, Western blot analysis of e11.5 DSCAM ${ }^{-1-}$ spinal cords reveal that DCC and Neogenin expression was maintained at levels comparable to wild-type ( $n=3$ per genotype).

\section{Results \\ Normal commissural axon trajectory in DSCAM mutant spinal cord}

The recent generation of DSCAM-null mice afforded us the opportunity to test genetically whether DSCAM is required for normal $\mathrm{C}$ axon guidance. DSCAM deletion of exon 1 results in complete ablation of DSCAM mRNA as assessed by in situ hybridization and Northern blotting, providing evidence that this allele is a null mutation of DSCAM $\left(\right.$ DSCAM $^{-/-}$) (Amano et al., 2009). When maintained on a Bl6 background the majority of $D S C A M^{-/-}$animals were reported to die within $48 \mathrm{~h}$ of birth due to respiratory deficiencies (Amano et al., 2009) which we also observed (9 litters analyzed, 15 DSCAM mutants; data not shown). To investigate the effect of complete DSCAM ablation on $\mathrm{C}$ axon guidance, we monitored $\mathrm{C}$ axon projections by TAG-1 immunostaining in lumbar, thoracic and cervical spinal cord sections of embryonic day 11.5 (e11.5) DSCAM ${ }^{-1-}$ mutants and control wild-type littermates (Dodd et al., 1988; Amano et al., 2009). In DSCAM-null spinal cords, TAG-1 protein expression was detected in a similar way in $C$ neurons, when compared with control littermates, and, surprisingly, TAG-1-expressing $\mathrm{C}$ axons reached and crossed the floor plate as in control littermates (Fig. $1 A-C, E-G ; n=4$ embryos per genotype). To ascertain whether an earlier $\mathrm{C}$ axon projection phenotype might be corrected by e11.5, we also examined e10.5 TAG-1-expressing $\mathrm{C}$ axons in spinal cord sections but found no differences between wild-type and $D S C A M^{-1-}$ embryos (Fig. $1 D, H ; n=4$ embryos per genotype). We also examined the development of corpus callosum, hippocampal, anterior and posterior commissures whose formation is netrin-dependent (Serafini, 1996). We did not observe any obvious defects in the development of these structures in e18.5 DSCAM-null mutant embryos (data not shown).

To rule out the possibility of compensation by increased expression of other Netrin-1 receptors, we examined the expression of DCC and Neogenin in DSCAM ${ }^{-1-}$ and control embryos at e10.5 and e11.5, when $\mathrm{C}$ axons are reaching and crossing the floor plate (Dodd et al., 1988). Analysis of $D S C A M^{-/-}$e11.5 spinal cords revealed the absence of DSCAM mRNA (Fig. 1M) and expression of DCC and Neogenin mRNA and protein at levels comparable to wild-type littermate (Fig. $1 \mathrm{~J}, \mathrm{~K}, \mathrm{~N}, \mathrm{O}, \mathrm{Q}$; data not shown; $76.2 \pm 18.5$ vs $83 \pm 8.5$ arbitrary units wt vs $D S C A M^{-/-}$ DCC protein, respectively; $p>0.5$ Student's $t$ test; $109.1 \pm 7$ vs $99.23 \pm 0.4$ arbitrary units wt vs $D S C A M^{-/-}$Neogenin protein, respectively; $p=0.237$ Student's $t$ test).

\section{Netrin-1-induced axon outgrowth is unaffected by DSCAM mutation}

We reasoned that the lack of $\mathrm{C}$ axon projection phenotypes in $D S C A M^{-1-}$ spinal cords could be because of partial compensation by cues other than Netrin-1 (Charron et al., 2003; Evans and Bashaw, 2010) or because of a very high endogenous Netrin-1 concentration (Serafini et al., 1994; Moore and Kennedy, 2006). To isolate the effect of Netrin-1 in C axon outgrowth and to better control its concentration, dorsal spinal cords explants of e11.5 DSCAM ${ }^{-/-}$mutants and control littermates were cultured in the presence or absence of Netrin-1 at a concentration previously shown to be sensitive to the loss of $\mathrm{Ne}$ - 
trin receptor function (Fig. 2) (Serafini et al., 1994; Moore and Kennedy, 2006). In DSCAM ${ }^{-1-}$ and control explants, axonal outgrowth was only detected in the presence of $200 \mathrm{ng} / \mathrm{ml}$ Netrin-1 (Fig. 2D-F). Moreover, in DSCAM mutants the mean length of axon bundles was $42.8 \%$ of explant circumference and was not significantly different from that observed in DSCAM heterozygous or wild-type littermates (Fig. $2 G$; $51.8 \%$ and $47.6 \%$ respectively, $p=0.48 D^{2} C A M^{+/-}$vs $D S C A M^{-/-}, p=0.76$ wild-type vs $D_{S C A M}{ }^{-1-}$, Student's $t$ test). These data demonstrate that DSCAM is dispensable for the in vitro outgrowth of $\mathrm{C}$ axons in response to Netrin-1.

Normal commissural axon floor plate crossing in DSCAM-null mutants Once $\mathrm{C}$ axons reach the floor plate, they cross it, enter one of the longitudinal fascicles on the contralateral side and project rostrally. To determine whether any of these stereotyped events were affected in DSCAM mutants we labeled C axons by injecting DiI into the dorsal spinal cord of fixed e12.5 embryos at several rostrocaudal locations and, to allow the dye time to diffuse, we analyzed the location of $\mathrm{C}$ axons $24 \mathrm{~h}$ later. In wild-type embryos, DiI-labeled $\mathrm{C}$ axons reached the floor plate, where only $24 \%$ of them were stalled, while a majority of them were observed to project rostrally (76\%). Analysis of a similar number of injection sites in DSCAM ${ }^{-1-}$ mutants showed 22\% of $\mathrm{C}$ axons were stalled at the floor plate while $78 \%$ of them were found to project anteriorly after crossing the floor plate, indicating normal projections of commissural neurons in the absence of DSCAM $\left(p=0.82, \chi^{2}\right.$ test for independence; Fig. 3C).

\section{Absence of DSCAM does not enhance the commissural axon trajectory defects in DCC mutants}

DSCAM was shown to interact with the Netrin-1 receptor DCC (Ly et al., 2008; Liu et al., 2009). Since in DCC mutants a small percentage of $\mathrm{C}$ axons is able to reach and cross the floor plate (Keino-Masu et al., 1996) we reasoned that in this C axon subpopulation, DSCAM might be required for axonal attraction toward the floor plate. We therefore examined $\mathrm{C}$ axon trajectory by TAG-1 immunostaining in e11.5 brachial spinal cords of $D C C^{-/-}$ $D S C A M^{-/-}$compound mutants, $D C C^{-/-}$mutants and control littermates. In $D C C^{-/-}$; DSCAM ${ }^{-/-}$compound mutants the majority of TAG-1 C axons were not able to reach the floor plate, and surprisingly, similar number of $\mathrm{C}$ axons reached and crossed the floor plate as observed in DCC mutants (Fig. $4 C, D ; n=3 \mathrm{DCC}^{-/-} ; \mathrm{DSCAM} \mathrm{M}^{-/-}$and $3 \mathrm{DCC}^{-/-}$embryos),
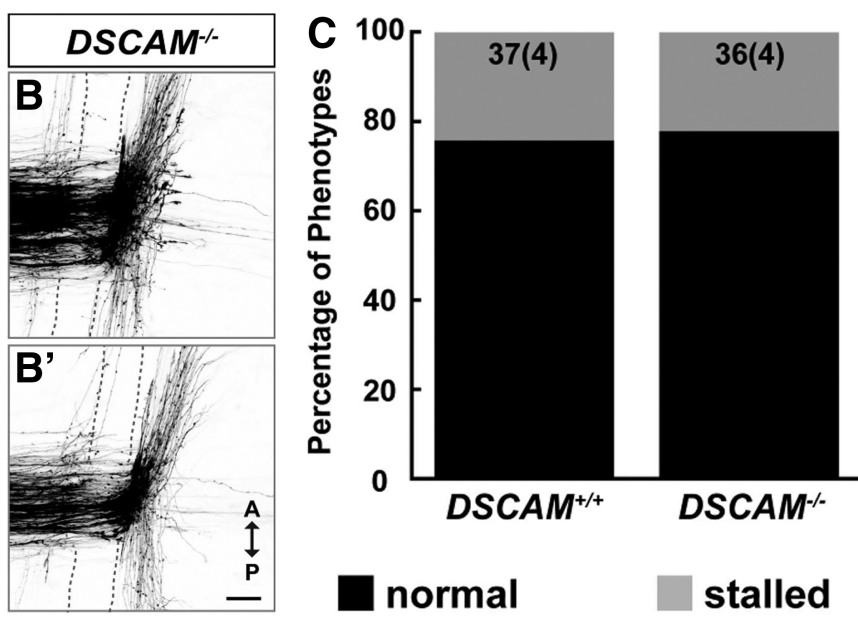

normal

stalled

Figure 3. Normal commissural axon floor plate crossing in DSCAM-null mutants. $\boldsymbol{A}-\boldsymbol{B}$, Dil injections into e12.5 dorsal spinal axons crossed the floor plate (dashed line) and turned anteriorly. $C$, Quantification of Dil labeled axonal projections. $N=36$ injections in DSCAM ${ }^{-1-}$ and 37 in wild-type embryos; $n=4$ embryos per genotype. Scale bar (in $\boldsymbol{B}^{\prime}$ ), $50 \mu \mathrm{m}$ in all panels.

\section{DCC $+/ / D S C A M^{+/+}$}
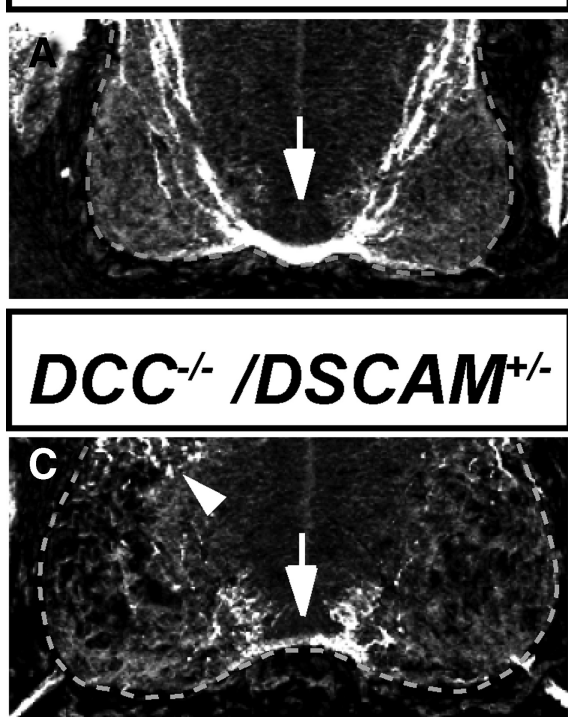

\section{$D_{C} C^{+/ /} / D S C A M^{-/}$}
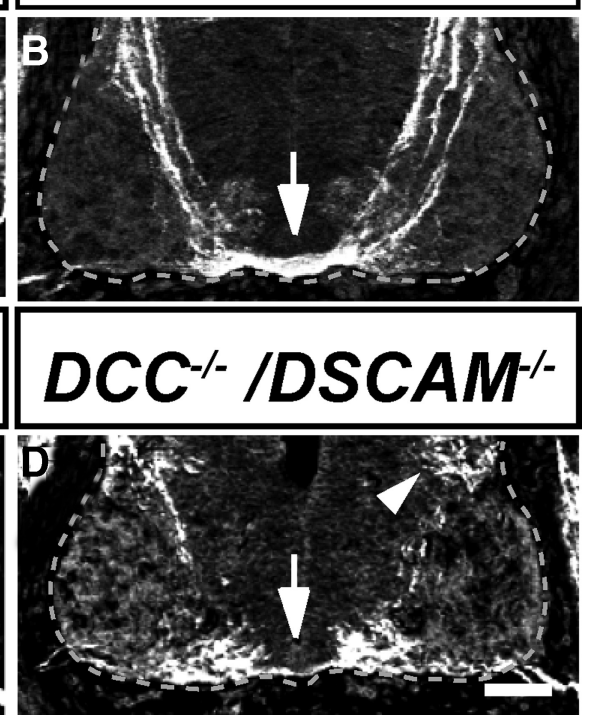

Figure 4. $D C C / D S C A M$ compound mutants have commissural axon trajectory similar to $D C C$ mutants. $A-D, T A G-1$ immunostaining at e11.5 brachial spinal cord embryos. Commissural neurons of $D C C^{+/-} ; D S C A M^{-1-}$ embryos $(\boldsymbol{B})$ reached and crossed the floor plate as in control littermates $(\boldsymbol{A})$. Like $D C C$ mutants $(\boldsymbol{C})$, a small percentage of TAG-1 commissural neurons of e11.5 DCCOSCAM compound mutant embryos (D) reached and crossed the floor plate (arrows) while the majority were not able to reach the floor plate (arrowheads). $N=3 \mathrm{DCC}^{-/-} ; \mathrm{DSCAM}^{-/-}, 3 \mathrm{DCC}^{-/-}, 3 \mathrm{DCC}^{+/-} ; \mathrm{DSCAM}^{-/-}$, and 3 control embryos. Scale bar, $50 \mu \mathrm{m}$ in all panels.

indicating that the lack of DSCAM expression does not exacerbate the $D C C^{-/-} \mathrm{C}$ axon phenotype. We next asked whether DSCAM loss could be exacerbated by the loss of one allele of DCC and examined C axon trajectories in e 11.5 brachial spinal cords of $D C C^{+/-}$; DSCAM ${ }^{-/-}$mutants. In these, we observed a similar number of $\mathrm{C}$ axons reaching and crossing the floor plate as in control animals (Fig. $4 A, B ; n=3$ per genotype).

\section{Discussion}

Our observations indicate that in mice, DSCAM is not required for Netrin-induced axon outgrowth ex-vivo or for guidance to and at the floor plate in vivo. Here we discuss the differences between genetic 
and RNA interference-mediated loss of DSCAM function and possible compensatory mechanisms in Netrin signaling.

The main published evidence for DSCAM function as Netrin receptor in vertebrates comes from acute downregulation of DSCAM using specific siRNAs, resulting in defects of Netrin-induced commissural axon outgrowth and guidance to the midline (Ly et al., 2008; Liu et al., 2009). These experiments also included well controlled rescue experiments arguing that the effects of DSCAM loss are specific. Generally, siRNAs constitute a useful tool for functional analysis and their effects recapitulate phenotypes observed in mice null mutants (Helmbacher et al., 2000; Luria et al., 2008; Kao et al., 2009; Palmesino et al., 2010). However, this is not always the case as it was previously reported that some siRNA phenotypes were not confirmed by genetic analyses in mice ( $\mathrm{Wu}$ et al., 2006; Moore et al., 2008). These and our observations suggest that acute loss of gene function may differ in its effects from a deletion of function over a long term, such as a null genetic mutation.

The divergences in phenotype between DSCAM downregulation through siRNA and complete genetic ablation can also be explained by compensatory effects, in which acute downregulation of DSCAM does not allow sufficient time for other components of Netrin-1 signaling to compensate for the lack of DSCAM expression. However, in DSCAM mutants, expression of other known Netrin-1 receptors was unaffected, suggesting that, if it does occur, the lack of DSCAM expression may be corrected in more subtle ways. Possible compensatory mechanisms may occur in the post-translational regulation of other Netrin receptors or in the regulation of downstream signaling such as DCC phosphorylation (Meriane et al., 2004). Though we cannot exclude this possibility completely, the observation that mice lacking both DCC and DSCAM have C axon guidance errors similar to what is observed in mice lacking only DCC (Keino-Masu et al., 1996) argue against it.

Netrin-1 is secreted by floor plate cells and forms a gradient in the spinal cord (Kennedy et al., 2006) and thereby induces first, axon outgrowth, and then guidance of the axons to the midline (Serafini et al., 1994; Ming et al., 2002). Maximal outgrowth of dorsal spinal cord explants happens in the presence of $200 \mathrm{ng} / \mathrm{ml}$ Netrin-1 (Serafini et al., 1994; Moore and Kennedy, 2006). Our results show that in the presence of this optimal concentration of Netrin, C axons of DSCAM mutants extend their axons to levels comparable to wild-type embryos. It cannot be excluded that DSCAM may determine the sensitivity at which $\mathrm{C}$ axons are able to respond to Netrin-1, but, if so, this clearly is not sufficient to produce in vivo defects.

Could other axonal projections be more sensitive to loss of DSCAM than commissural neurons? In addition to $\mathrm{C}$ neurons, Netrin-1 plays a role in axon guidance for many other neuronal types and where examined, these neurons are known to require DCC to respond to Netrin-1 (Moore, 2007). We cannot, however, exclude the possibility that DSCAM is required for responses of some neuronal populations to Netrin-1. In this context, it is interesting to note that DSCAM mutants display abnormal Botzinger complex rhythmicity (Amano et al., 2009), which is dependent on normal hindbrain commissure development. In addition, DSCAM might have Netrin-1 independent functions, as suggested by the observation that DSCAM is required for retinal ganglion cell dendrite fasciculation (data not shown; Fuerst et al., 2009).

In summary, based on the absence of axon outgrowth and axon guidance defects in DSCAM-null mice, the lack of compen- sation by expression of other Netrin receptors and the lack of enhanced guidance defects in DCC/DSCAM mutants compared with DCC-null mice, we conclude that DSCAM is not required for Netrin-1-mediated C axon guidance in vertebrates.

\section{Note added in proof}

Using the described conditions, we could not see any difference in DSCAM expression in spinal cord sections of wild-type and DSCAM mutants using five different commercial antibodies: mouse anti-DSCAM (Millipore), rabbit anti-DSCAM (Novus), and goat anti-DSCAM (R\&D Systems) used at a 1:100 dilution.

\section{References}

Amano K, Fujii M, Arata S, Tojima T, Ogawa M, Morita N, Shimohata A, Furuichi T, Itohara S, Kamiguchi H, Korenberg JR, Arata A, Yamakawa K (2009) DSCAM deficiency causes loss of pre-inspiratory neuron synchroneity and perinatal death. J Neurosci 29:2984-2996.

Andrews GL, Tanglao S, Farmer WT, Morin S, Brotman S, Berberoglu MA, Price H, Fernandez GC, Mastick GS, Charron F, Kidd T (2008) Dscam guides embryonic axons by Netrin-dependent and -independent functions. Development 135:3839-3848.

Charron F, Stein E, Jeong J, McMahon AP, Tessier-Lavigne M (2003) The morphogen sonic hedgehog is an axonal chemoattractant that collaborates with netrin-1 in midline axon guidance. Cell 113:11-23.

Dodd J, Morton SB, Karagogeos D, Yamamoto M, Jessell TM (1988) Spatial regulation of axonal glycoprotein expression on subsets of embryonic spinal neurons. Neuron 1:105-116.

Evans TA, Bashaw GJ (2010) Axon guidance at the midline: of mice and flies. Curr Opin Neurobiol 20:79-85.

Fazeli A, Dickinson SL, Hermiston ML, Tighe RV, Steen RG, Small CG, Stoeckli ET, Keino-Masu K, Masu M, Rayburn H, Simons J, Bronson RT, Gordon JI, Tessier-Lavigne M, Weinberg RA (1997) Phenotype of mice lacking functional Deleted in colorectal cancer (Dcc) gene. Nature 386:796-804.

Fuerst PG, Bruce F, Tian M, Wei W, Elstrott J, Feller MB, Erskine L, Singer JH, Burgess RW (2009) DSCAM and DSCAML1 function in self-avoidance in multiple cell types in the developing mouse retina. Neuron 64:484-497.

Helmbacher F, Schneider-Maunoury S, Topilko P, Tiret L, Charnay P (2000) Targeting of the EphA4 tyrosine kinase receptor affects dorsal/ventral pathfinding of limb motor axons. Development 127:3313-3324.

Kao TJ, Palmesino E, Kania A (2009) SRC family kinases are required for limb trajectory selection by spinal motor axons. J Neurosci 29:5690-5700.

Keino-Masu K, Masu M, Hinck L, Leonardo ED, Chan SS, Culotti JG, TessierLavigne M (1996) Deleted in Colorectal Cancer (DCC) encodes a netrin receptor. Cell 87:175-185.

Kennedy TE, Wang H, Marshall W, Tessier-Lavigne M (2006) Axon guidance by diffusible chemoattractants: a gradient of netrin protein in the developing spinal cord. J Neurosci 26:8866-8874.

Liu G, Li W, Wang L, Kar A, Guan KL, Rao Y, Wu JY (2009) DSCAM functions as a netrin receptor in commissural axon pathfinding. Proc Natl Acad Sci U S A 106:2951-2956.

Luria V, Krawchuk D, Jessell TM, Laufer E, Kania A (2008) Specification of motor axon trajectory by ephrin-B:EphB signaling: symmetrical control of axonal patterning in the developing limb. Neuron 60:1039-1053.

Ly A, Nikolaev A, Suresh G, Zheng Y, Tessier-Lavigne M, Stein E (2008) DSCAM is a netrin receptor that collaborates with DCC in mediating turning responses to netrin-1. Cell 133:1241-1254.

Meriane M, Tcherkezian J, Webber CA, Danek EI, Triki I, McFarlane S, Bloch-Gallego E, Lamarche-Vane N (2004) Phosphorylation of DCC by Fyn mediates Netrin-1 signaling in growth cone guidance. J Cell Biol 167:687-698.

Ming GL, Wong ST, Henley J, Yuan XB, Song HJ, Spitzer NC, Poo MM (2002) Adaptation in the chemotactic guidance of nerve growth cones. Nature 417:411-418.

Moore SW, Kennedy TE (2006) Protein kinase A regulates the sensitivity of spinal commissural axon turning to netrin-1 but does not switch between chemoattraction and chemorepulsion. J Neurosci 26:2419-2423. 
Moore SW, Tessier-Lavigne M, Keneny TE (2007) Netrins and their receptors. Adv Exp Med Biol 621:17-31.

Moore SW, Lai Wing Sun K, Xie F, Barker PA, Conti M, Kennedy TE (2008) Soluble adenylyl cyclase is not required for axon guidance to netrin-1. J Neurosci 28:3920-3924.

Palmesino E, Rousso DL, Kao TJ, Klar A, Laufer E, Uemura O, Okamoto H, Novitch BG, Kania A (2010) Foxp1 and lhxl coordinate motor neuron migration with axon trajectory choice by gating Reelin signalling. PLoS Biol 8:e1000446.

Schmucker D, Chen B (2009) Dscam and DSCAM: complex genes in simple animals, complex animals yet simple genes. Genes Dev 23:147-156.

Serafini T, Kennedy TE, Galko MJ, Mirzayan C, Jessell TM, Tessier-Lavigne M (1994) The netrins define a family of axon outgrowth-promoting proteins homologous to C. elegans UNC-6. Cell 78:409-424.
Serafini T, Colamarino SA, Leonardo ED, Wang H, Beddington R, Skarnes WC, Tessier-Lavigne M (1996) Netrin-1 is required for commissural axon guidance in the developing vertebrate nervous system. Cell 87:1001-1014.

Tessier-Lavigne M, Placzek M, Lumsden AG, Dodd J, Jessell TM (1988) Chemotropic guidance of developing axons in the mammalian central nervous system. Nature 336:775-778.

Tsuchida T, Ensini M, Morton SB, Baldassare M, Edlund T, Jessell TM, Pfaff SL (1994) Topographic organization of embryonic motor neurons defined by expression of LIM homeobox genes. Cell 79: 957-970.

Wu KY, Zippin JH, Huron DR, Kamenetsky M, Hengst U, Buck J, Levin LR, Jaffrey SR (2006) Soluble adenylyl cyclase is required for netrin-1 signaling in nerve growth cones. Nat Neurosci 9:1257-1264. 